[7] N. Sibony, Prolongement des fonctions holomorphes bornées et métrique de Carathéodory, Invent Math. 29 (1975), 205-230.

[8] J.Siciak, Separately analytic functions and envelopes of holomorphy of some lower dimensional subsets of C", Ann. Polon. Math. 22 (1969), 154-171.

[9] - Weak analytic continuation from compact subsets of $C^{n}$, in: Lecture Notes in Math. 364, Springer, 1974, 92-96.

10] L. Waelbroeck, Weak analytic functions and the closed graph theorem, ibid., 97-100.

[11] V. P. Za kharyuta, Separately analytic functions, generalizations of the Hartogs theorem and envelopes of holomorphy, Mat. Sb. 101 (143) (1) (1976), 57.76 (in Russian).

DEPARTMENT OF MATHEMATICS

HANOI PEDAGOGICAL INSTITUTE

Hanoi, Vietnam

Received October 12, 1988

\section{Applications du théorème de factorisation pour des fonctions à valeurs opérateurs}

par

QUANFUA XU (Wuhan et Paris)

Alstract. In this paper we prove a factorization theorem for analytic functions with values in noncommutative $L_{p}$-spaces associated with a semifinite von Neumann algebra. Then we give two applications. The first is to show the Halagerup noncommutative $L_{p}$-spaces $(0<p<1)$ associated with an arbitrary von Neumann algebra are uniformly Hardy convex. In particular, they have the analytic Radon-Nikodym property. The second is to compute the interpolation spaces between the Hardy spaces of functions with values in noncommutative $L_{p}$-spaces associated with a semifinite von Neumann algebra.

$\S 1$. Introduction. On étudie dans cet article la convexité uniforme complexe des espaces non-commutatifs $L_{p}(M, \tau)(0<p<1)$ associés à une algèbre de von Neumann $M$, munie d'une trace normale semi-finie et fidèle $\tau$; on calcule aussi les espaces d'interpolation complexes entre les espaces de Hardy de fonctions à valeurs dans $L_{p}(M, \tau)$. L'outil que l'on utilise est des théorèmes de factorisation pour des fonctions à valeurs opérateurs. Le problème de factoriser une fonction en certaines composantes canoniques est bien classique. On présente, pour notre but, une forme de ces théorèmes. Notre théorème de factorisation concerne la factorisation pour des fonctions dans les espaces de Hardy à valeurs dans $L_{p}(M, \tau)$. Il se comporte presque comme le théorème classique de factorisation pour des fonctions scalaires dans les espaces de Hardy usuels. On l'applique ensuite pour démontrer que $L_{p}(M, \tau)(0<p<1)$ est uniformément convexe complexe en un certain sens. On en déduit, en particulier, que $L_{p}(M, \tau)(0<p<1)$ a la propriété de Radon-Nikodym analytique (en bref, ARNP). La deuxième application de ce théorème de factorisation est le calcul des espaces dinterpolation complexes entre les espaces de Hardy de fonctions à valeurs dans $L_{p}(M, \tau)(0<p<\infty)$. On démontre que pour $0<p_{0}, p_{1}, q_{0}, q_{1}<\infty, 0<\theta<1$,

$$
\left(H_{p_{0}}\left(L_{q_{10}}(M, \tau)\right), H_{p_{1}}\left(L_{q 1}(M, \tau)\right)\right)_{\theta}=H_{p}\left(L_{q}(M, \tau)\right),
$$

où $1 / p=(1-\theta) / p_{0}+\theta / p_{1}, 1 / q=(1-\theta) / q_{0}+\theta / q_{1}$.

On présente le théorème de factorisation dans le deuxième paragraphe. Nous basons notre démonstration sur un théorème classique de factorisation pour des fonctions à valeurs opérateurs, dû à Devinatz [D]. 
On sait que les espaces $L_{n}(0<p<1)$ associés à un espace mesu arbitraire ont ARNP. Il est naturel de demander si le même résultat est aus vrai dans le cas non-commutatif, i.e., si $L_{p}(M, \tau)(0<p<1)$ a aussi ARN (Kalton $[\mathrm{K}]$ a posé ce problème pour les classes de Schatten $C_{m}$, i.e., les espaci non-commutatifs $L_{p}\left(B\left(l_{2}\right), \operatorname{tr}\right)$ avec la trace usuelle tr). On répond positivemer au problème dans le troisième paragraphe. Le résultat est en fait ur conséquence immédiate d'un théorème plus fort: $L_{p}(M, \tau)(0<p<1)$ o uniformément convexe de Hardy (voir l'explication de la notion dans le texte En remarquant que les espaces $L_{n}(M, \tau)(1<p<\infty)$ sont uniformémes convexes réels (une propriété beaucoup plus forte que les deux mentionné ci-dessus), pour démontrer la convexité uniformo complexe de $L_{p}(M$, $(0<p<1)$ on utilise le théorème de factorisation pour ramener le cas $p<$ à celui de $p>1$. Plus généralement, on demontre que l'espace non-commuta $L_{p}$ de Haagerup $(0<p<1)$ associé à une algèbre de von Neumann arbitrai (pas nécessairement semi-finie) est aussi uniformément convexe de Hardy.

Dans le dernier paragraphe on calcule explicitement les espaces d'inté polation complexes entre les espaces de Hardy de fonctions a valeurs da $L_{p}(M, \tau)$. Le fait suivant est bien connu: si tous les indices dans (1.1) sol supérieurs à $1,(1.1)$ est alors vrai (car dans ce cas-là, lá projection de Riesz e bornée dans $L_{p}\left(L_{q}(M, \tau)\right)$ pour $1<p, q<\infty$, cf. [BGM]). En utilisant théorème de factorisation, on réduit tous les cas restant à celui-là. Dans , paragraphe, on démontre aussi que l'espace d'interpolation d'indice $\theta$ ent $L_{q 0}(M, \tau)$ et $L_{q}(M, \tau)\left(0<q_{0}<1,0<q_{1} \leqslant \infty\right)$ est exactement $L_{q}(M, \tau)$ av $1 / q=(1-0) / q_{0}+\theta / q_{1}$. La démonstration utilise encore le théoreme de fa torisation pour ramener le cas général au cas où tous les indices sont supérieu. ou égaux à 1 (dans ce cas-là, le résultat est connu).

L'auteur tient à remercier le Professeur Gilles Pisier pour plusieu discussions précieuses et stimulantes et aussi pour son encouragement penda la préparation du travail. 11 remercie également le Professeur Uffe Haageru pour l'avoir informé du résultat de [H2].

§ 2. Préliminaires et théorème de factorisation. Les espaces quasi-norm considérés dans cet article seront tous sur le corps des complexes et continu i.e., leurs quasi-normes sont uniformément continues sur leurs boules unité

Soit $(X,\|\cdot\|)$ un tel espace. Notons par $D$ le disque unité ouvert et $T$ cercle unité. $T$ s'identifie aussi à l'intervalle $[0,2 \pi]$. Soit $(\Omega, \Sigma, \mu)$ un espar mesuré. On note par $L_{p}(X, \mu)(0<p \leqslant \infty)$ l'espace des fonctions forteme mesurables par rapport à $\Sigma$ à valeurs dans $X$ telles que

$$
\|f\|_{p}=\left(\int\|f(\omega)\|^{n} d \mu((\omega))\right)^{1 / p}<\infty
$$

(avec la convention habituelle pour $p=\infty$ ). Soit $0<p<\infty$. Notons par $H_{p}(2$ le sous-espace fermé de $L_{p}(X, T, d t /(2 \pi))$ engendré par les polynômes trigonom triques analytiques $P$ à valeurs dans $X$, i.e., les polynômes $P$ de la forme suivant

$$
P(z)=\sum_{k=0}^{n} x_{k} z^{k} \quad(z \in T)
$$

pour un certain $n \in N$ et une suite finie $\left(x_{k}\right)$ dans $X . H_{\infty}(X)$ est le sous-espace fermé de $L_{x \rightarrow}(X, T, d t /(2 \pi))$ composé des fonctions dont les spectres des séries de Fourier formelles sont contenus dans $N$.

Une autre classe de Hardy que l'on utilisera est celle des fonctions analytiques sur le disque unité. Une fonction $f: D \rightarrow X$ est dite analytique dans $D$ si

$$
f(z)=\sum_{n \rightarrow 0}^{(x)} x_{n} z^{n}, \quad x_{n} \in X(n \geqslant 0), z \in D,
$$

où la série converge uniformément sur tout compact de $D$.

Définissons, pour $0<p<\infty$,

$$
\|f\|_{H_{N}^{\prime \prime}(x)}=\sup _{0<r<1}\left(\int_{0}^{2 \pi}\left\|f\left(r e^{i t}\right)\right\|^{p} \frac{d t}{2 \pi}\right)^{1 / p}
$$

Posons

$$
H_{p}^{D}(X)=\left\{f: D \rightarrow X \text { analytique: }\|f\|_{H_{D}^{D}(X)}<\infty\right\} .
$$

$H_{\infty}^{D}(X)$ désigne l'espace des fonctions analytiques bornées dans $D$ à valeurs dans $X$.

$M$ désignera toujours, sauf mention du contraire, une algèbre de von Neumann agissant sur un espace de Hilbert complexe $H$ et possédant une trace $\tau$ normale, semi-finie et fidele. $B(H)$ désignera l'espace des opérateurs bornés sur $H$, muni de la norme d'opérateurs. Soit $a$ un opérateur mesurable sur $H$ (il sera toujours sous-entendu que a est mesurable par rapport à $(M, \tau)$ au sens de Nelson [N]). Posons, pour $0<p<\infty$,

$$
\|a\|_{p}=\left[\tau\left(|a|^{p}\right)\right]^{1 / p}
$$

où $|a|$ est la valeur absolue de $a$. L'espace $L_{p}(M, \tau)$ se compose de tous les opérateurs mesurables $a$ tels que $\|a\|_{p}<\infty$. M est aussi noté par $L_{\infty x}(M, \tau)$. Il est connu que $\left\|^{*}\right\|_{p}$ est une norme (resp. $p$-norme) sur $L_{p}(M, \tau)$ pour $1 \leqslant p<\infty$ (resp. $0<p<1$ ) et que $L_{p}(M, \tau)$ est complet par rapport à $\|\cdot\|_{p} . M_{p}$ désignera la famille des projections dans $M$. Une projection $e \in M$ est dite de rang fini (par rapport à $\tau$ ) si $\tau(c)<\infty$. Un opérateur $a \in M$ est dit de rang fini si supp $(a)$ (la plus petite projection $e \in M$ telle que $a e=a$ ) est de rang fini. La famille $F(M)$ de tous les opérateurs de rang fini dàns $M$ est dense dans $L_{p}(M, \tau)(0<p<\infty)$. Désignons par $A F^{\prime}(M)$ la famille des fonctions y dans $H_{x, 1}\left(L_{* x}(M, \tau)\right)$ telles que $\operatorname{supp}(f(z))$ soit contenu dans une projection fix de de rang fini pour presque tout $z \in T$. Alors $A F(M)$ est dense dans $H_{p}\left(L_{q}(M, \tau)\right)$ pour $0<p, q<\infty$.

On utilisera aussi une autre formule de la norme dans $L_{p}(M, \tau)$ d'un opérateur mesurable $a$, définie par les s-nombres généralisés de $a$. Soit $0<t<\infty$. Let $t$-ic̀me s-nombre gúnéralisé de $a$ est le nombre

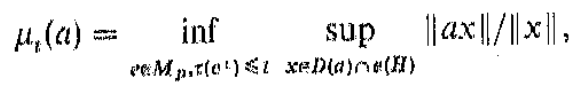


où $e^{\perp}=1-e$ et où $D(a)$ est le domaine de définition de $a$. Il est alors facile de vérifier que

$$
\|a\|_{p}=\left(\int_{0}^{\infty} \mu_{t}(a)^{p} d t\right)^{1 / p}
$$

On vérifie aussi facilement que $a$ est borné si et seulement si $\mu_{t}(a)$ est borné et que

$$
\|a\|=\lim _{t \rightarrow 0^{+}} \mu_{t}(a)
$$

que $a$ est de rang fini si et seulement si $\mu_{t}(a)$ s'annule pour $t$ suffisamment grand et que

$$
\tau(\operatorname{supp}(a))=\inf \left\{t>0: \mu_{t}(a)=0\right\} .
$$

Tout ce que l'on vient de dire sur $L_{p}(M, \tau)$ non-commutatif associé à une algébre de von Neumann semi-finie est la généralisation naturelle de $C_{k}$ (associé à la trace usuelle sur $B\left(l_{2}\right)$ ). On envoie le lecteur, e.g., à $[F K],[N]$, [O]: [Se] pour plus d'information.

Le théorème de factorisation que l'on va utiliser dans les deux paragraphes suivants est le

ThÉORÈMe 2.1. Soient $0<p, q<\infty$. Soient $0<p_{0}, p_{1}, q_{0}, q_{1}<\infty$ tels quc $1 / p=1 / p_{0}+1 / p_{1}, 1 / q=1 / q_{0}+1 / q_{1}$. Alors pour toute fonction $f \in A F(M)$ et toul $\varepsilon>0$, il existe deux fonctions $g, h \in A F(M)$ telles que $f=g h$ et

$$
\|g\|_{H_{p_{0}}\left(L_{q_{0}}(M, \tau)\right)}\|h\|_{H_{p_{1}}\left(L_{q_{1}}(M, \tau)\right)} \leqslant\|f\|_{H_{p}\left(L_{q}(M, \tau)\right)}+\varepsilon
$$

Remarques. (i) Le théorème 2.1 est essentiellement connu dans le cas oì $M=B\left(l_{2}\right)$ et $\tau$ est la trace usuelle, i.e., dans le cas $C_{p}$ (cf. [S], [D]).

(ii) Si $f=g h$, on a de plus, par l'inégalité de Hölder,

$$
\|f\|_{H_{p}\left(L_{q}(M, \tau)\right)} \leqslant\|g\|_{H_{p_{0}}\left(L_{q_{0}}(M, \tau)\right)}\|h\|_{H_{p_{1}}\left(L_{q_{1}}(M, \tau)\right)} \text {. }
$$

Donc le théorème 2.1 dit grossièrement que toute fonction dans $H_{p}\left(L_{q}(M, \tau)\right.$ peut s'écrire comme un produit de deux fonctions dans $H_{p 0}\left(L_{q_{0}}(M, \tau)\right)$ $H_{p_{1}}\left(L_{q_{1}}(M, \tau)\right)$ respectivement et aussi leurs normes.

L'idée de la démonstration suivante du théorème est dans un exposé de Gilles Pisier sur le même sujet. Pour simplifier la notation, on notera la norme de $H_{p}\left(L_{q}(M, \tau)\right)$ par $\|\cdot\|_{p q}$ ou simplement par $\|\cdot\|_{p}$ si $p=q$. Commençons par ls lemme suivant, qui est même "plus précis" que le théorème lui-même.

LEMME 2.2. Supposons que $\tau$ est finie, i.e., $\tau(1)<\infty$. Soit $0<\lambda<1$. Soien $f \in A F(M)$ et $\varepsilon>0$. Il existe alors deux fonctions $g, h \in A F(M)$ telles qu $f(z)=g(z) h(z)$ pour presque tout $z \in T$ et

$$
\begin{aligned}
& \mu_{t}(g(z)) \leqslant\left(\mu_{t}(f(z))\right)^{1-\lambda}+\varepsilon \quad(t>0) . \\
& \mu_{t}(h(z)) \leqslant\left(\mu_{t}(f(z))\right)^{\lambda}+\varepsilon \quad
\end{aligned}
$$

Démonstration. Soit $\delta>0$ arbitrairement fixé. Posons

$$
w(z)=\left(\delta+f^{*}(z) f^{\prime}(z)\right)^{\lambda}, \quad z \in T .
$$

Alors

$$
\delta^{\lambda} \leqslant w(z) \leqslant\left(\delta+\|f\|_{\infty}^{2}\right)^{\lambda}, \quad \forall z \in T .
$$

$w(z)$ est un opérateur borné positif dans $M$ pour tout $z \in \boldsymbol{T}$. D'après un theorème classique de Devinatz (cf. [D], th. 3.2), il existe une fonction unique $\varphi \in H_{\infty}(B(H))$ telle que $\varphi^{-1}(z)$ existe p.p., $\varphi^{-1} \in H_{\infty}(B(H)), \hat{\varphi}(0)>0(\hat{\varphi}(n)$ étant le $n$-ième coefficient de Fourier de $\varphi, n \in Z$ ) et telle que

$$
w(z)=\varphi^{*}(z) \varphi(z) \quad \text { p.p. }(z \in T)
$$

On affirme que $\varphi(z) \in M$ p.p. En effet, pour un opérateur unitaire quelconque $u$ dans le commutant $M^{\prime}$ de $M, u \varphi u^{*}$ possède encore la même propriété ci-dessus que $\varphi$. L'unicité implique qu'alors $\varphi=u \varphi u^{*}$. D'où $\varphi$ commute à $u$. Cela démontre notre assertion. Donc $\varphi, \varphi^{-1} \in H_{\infty}\left(L_{\infty}(M, \tau)\right)$. Posons

$$
g=f \varphi^{-1}, \quad h=\varphi,
$$

Alors $g, h \in A F(M)$ et $f=g h$. Soit $z \in T$ tel que (2.7) soit vérifié. On a, pour $\forall t>0$,

$$
\mu_{t}^{2}(h(z))=\mu_{t}\left(\varphi^{*}(z) \varphi(z)\right)=\mu_{t}(w(z))=\mu_{t}^{2 \lambda}(f(z))+O(\delta) .
$$

Cela donne (2.6) en choisissant convenablement $\delta$ (en remarquant, d'après (2.2), (2.3), que $\mu_{t}(f(z))$ est uniformément borné en $t>0, z \in T$ et s'annule uniformément en $z \in T$ pour $t$ suffisamment grand pour toute fonction $f \in A F(M)$ ).

On va ensuite démontrer (2.5). Soit encore $z \in T$ tel que (2.7) soit vérifié. Soit $t>0$. On a

(2.8) $\quad \mu_{t}^{2}(g(z))=\mu_{i}\left(\varphi^{*-1}(z) f^{*}(z) f^{\prime}(z) \varphi^{-1}(z)\right)$

$$
\begin{aligned}
& =\inf \left\{\sup \left\langle f^{*}(z) f(z) \varphi^{-1}(z) x, \varphi^{-1}(z) x\right\rangle\right\}
\end{aligned}
$$

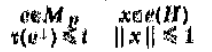

$$
\begin{aligned}
& =\inf \left\{\sup _{0 *}\left\langle f^{*}(z) f(z) x, x\right\rangle\right\} \quad \text { (d'après (2.7)). } \\
& \text { (e) }
\end{aligned}
$$

D'autre part, on a

(2.9) $\quad \mu_{t}\left(w^{-1 / 2}(z) f^{*}(z) f^{\prime}(z) w^{-1 / 2}(z)\right)$.

$$
\begin{aligned}
& =\inf \left\{\sup \left\langle f^{*}(z) f(z) w^{-\infty} l / 2(z) x, w^{\cdots 1 / 2}(z) x\right\rangle\right\}
\end{aligned}
$$

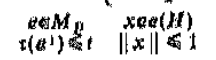

$$
\begin{aligned}
& \Rightarrow \inf \left\{\sup \left\langle f^{*}(z) f(z) x, x\right\rangle\right\} \text {. }
\end{aligned}
$$

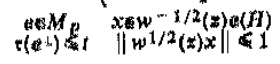


On affirme que

$$
\begin{aligned}
& \text { (2.10) } \quad \inf \left\{\sup _{z}\left\langle f^{*}(z) f(z) x, x\right\rangle\right\}
\end{aligned}
$$

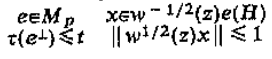

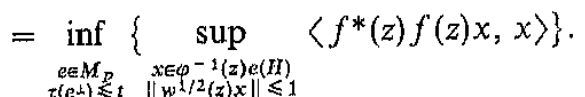

En effet, posons $a=\varphi^{*-1}(z) w^{1 / 2}(z)$. (2.7) montre que $a$ est unitaire. Donc pour $e \in M_{p}$, les projections $e, a e a^{*}$ sont équivalentes (au sens d'algèbres de von Neumann), d'où $\tau(e)=\tau\left(a e a^{*}\right)$. Or $\varphi^{-1}(z) a=w^{-1 / 2}(z)$ (d'après $(2.7)$ ). On en déduit facilement (2.10). Une combinaison de (2.8), (2.9) et (2.10) nous donne

$$
\begin{aligned}
\mu_{t}^{2}(g(z))=\mu_{t}\left(w^{-1 / 2}(z) f^{*}(z) f(z) w^{-1 / 2}(z)\right) & \leqslant\left[\mu_{t}\left(\delta+f^{*}(z) f(z)\right)\right]^{1-\lambda} \\
& =\left[\mu_{t}(f(z))\right]^{2(1-\lambda)}+O(\delta) .
\end{aligned}
$$

Ceci montre (2.5) en choisissant $\delta>0$ convenablement.

Corollaire 2.3. Supposons $\tau(1)<\infty$. Soient $0<q, q_{0}, q_{1}<\infty$ tels que $1 / q=1 / q_{0}+1 / q_{1}$. Pour tout $f \in A F(M)$ et tout $\varepsilon>0$, il existe $g, h \in A F(M)$ tels que $f(z)=g(z) h(z)$ et

$$
\left\{\begin{array}{l}
\|g(z)\|_{q_{0}} \leqslant\|f(z)\|_{q}^{(1-q) / q_{1}}+\varepsilon \quad \\
\|h(z)\|_{q_{1}} \leqslant\|f(z)\|_{q}^{q / q_{1}}+\varepsilon
\end{array} \quad \text { p.p. }(z \in T) .\right.
$$

Démonstration. Comme $\tau(1)<\infty$, pour $a \in M$, (2.1) et (2.3) nous donnent

$$
\|a\|_{p}=\left(\int_{0}^{\tau(1)} \mu_{t}(a)^{p} d t\right)^{1 / p} .
$$

Le corollaire résulte alors immédiatement du lemme 2.3 en prenant $\lambda=q / q_{1}$.

Remarque 2.4. Avec la même condition que dans le corollaire 2.3, si $F$ est une fonction dans $H_{x_{x}}^{D}(M)$ qui admet une limite radiale $f$ p.p. dans $M$ sur le cercle unité, alors $f \in A F(M)$. Soit $\varphi$ la fonction associée dans la démonstration du lemme $2.3\left(\lambda=q / q_{1}, \delta\right.$ étant fixé là-bas). Il existe alors une fonction $\Phi \in H_{\alpha,}^{D}(M)$ telle que

$$
\lim _{r \rightarrow 1} \Phi\left(r e^{i t}\right)=\varphi\left(e^{i t}\right) \quad \text { p.p. }
$$

pour la topologie forte d'opérateurs ( $\Phi$ étant l'intégrale de Poisson de $\varphi$ ); de plus, $\Phi^{-1} \in H_{\infty}^{D}(M), \quad \Phi(0)=\hat{\varphi}(0)>0$. Posons $G=F \Phi^{-1}, \quad H=\Phi$. Alors $G, H \in H_{\infty}^{D}(M), F=G H$ et

$$
\left\{\begin{array}{l}
\|G(z)\|_{q_{0}} \leqslant\|F(z)\|_{q}^{(1-q) / q_{1}}+\varepsilon \\
\|H(z)\|_{q_{1}} \leqslant\|F(z)\|_{q}^{q / q_{1}}+\varepsilon
\end{array} \quad \text { p.p. }(z \in T) .\right.
$$

Démonstration du théorème 2.1. Supposons d'abord que $\tau$ est finie. Soit $f \in A F(M)$ et soit $\varepsilon>0$. Posons $F=\varphi^{-1} f$, où $\varphi$ est la fonction extérieure telle que

$$
|\varphi(z)|=\|f(z)\|_{L_{q}(M, z)}+\delta, \quad z \in T,
$$

$\delta$ étant un nombre positif fixé. Alors $F \in A F(M)$ et $\|F\|_{\infty q q} \leqslant 1$. Soit $\varepsilon^{\prime}>0$, D'après le corollaire 2.3 , on trouve deux fonctions $G, H \in A F(M)$ telles que

$$
F=G H, \quad \sup \left(\|G\|_{\infty q_{0}},\|H\|_{\infty q_{1}}\right) \leqslant 1+\varepsilon^{\prime} .
$$

Posons $g=\varphi^{1-\mu} G, h=\varphi^{\mu} H$, où $\mu=p / p_{1}$. Alors $f=g h$ et de plus

$$
\|g\|_{p_{0 q} q_{0}} \leqslant\|G\|_{\infty q_{0}}\|\varphi\|_{p}^{1-\mu}, \quad\|h\|_{p_{1} q_{1}} \leqslant\|H\|_{\infty q_{1}}\|\varphi\|_{p}^{\mu} .
$$

D'où

$$
\|g\|_{p_{0 q} q}\|h\|_{p_{1 q} q_{1}} \leqslant\left(1+\varepsilon^{\prime}\right)\left(\|f\|_{p q}+O(\delta)\right),
$$

ce qui démontre (2.4) en choisissant convenablement $\varepsilon^{\prime}$ et $\delta$.

Passons maintenant au cas général. Soient $f \in A F(M)$ et $\varepsilon>0$. Il existe alors une projection $e \in M$ telle que $\tau(e)<\infty$ et telle que $f=e f e$. Notons par $M_{e}$ la restriction de $e M e$ sur $e(H)$, par $\tau_{e}$ la restriction de $\tau$ sur $M_{e} . \tau_{e}$ est donc finie. Par la démonstration précédente, il existe $\tilde{g}, \tilde{h} \in A F\left(M_{e}\right)$ tels que

$$
f_{e}=\tilde{g} \tilde{h}, \quad\|\tilde{g}\|_{p_{0} q_{0}}\|\tilde{h}\|_{p \times q_{1}} \leqslant\left\|f_{e}\right\|_{p q}+\varepsilon,
$$

où $f_{e}$ est la restriction de $f$ sur $e(H)$. Comme $e f e=f$, on a $\|f\|_{p q}=\left\|f_{e}\right\|_{p q}$. Posons

$$
g=\tilde{g} e, \quad h=\tilde{h e} .
$$

On vérifie aisément que $g, h \in A F(M), f=g h$ et que $g, h$ satisfont (2.4) (en remarquant que $\left.\|g\|_{p_{0} q_{0}}=\|\tilde{g}\|_{p_{0} q_{0}},\|h\|_{p_{1} q_{1}}=\|\tilde{h}\|_{p_{1} q_{1}}\right)$. On achève ainsi la démonstration du théorème.

Remarque. Après avoir démontré que $L_{p}(M, \tau)(0<p \leqslant 1)$ a ARNP dans le paragraphe suivant, on verra que le théorème 2.1 est aussi vrai si on remplace tous les $H_{p}\left(L_{q}(M, \tau)\right)$ par $H_{p}^{D}\left(L_{q}(M, \tau)\right)$.

§3. Convexité uniforme complexe. On va utiliser le théorème 2.1 pour démontrer le théorème suivant:

THÉRÈMe 3.1. Soit $0<p<1$. Il existe alors une constante positive $\alpha_{p}$ (ne dépendant que de $p$ ) telle que toute fonction $f \in H_{p}\left(L_{p}(M, \tau)\right)$ vérifie

(3.1) $\quad\left(\|f(0)\|_{L_{p}(M, \tau)}^{2}+\alpha_{p}\|f-\hat{f}(0)\|_{H_{p}\left(X_{p}(M, \tau)\right)}^{2}\right)^{1 / 2} \leqslant\|f\|_{H_{p}\left(L_{p}(M, \tau)\right)}$,

où $\hat{f}(0)$ est le terme constant de la série de Fourier formelle de $f$.

U. Haagerup et $\mathrm{G}$. Pisier ont démontré la même inégalité en remplaçant $L_{p}(M, \tau)$ par les duaux de $C^{*}$-algèbres (correspondant à $p=1$ dans (3.1), of $[\mathrm{HP}]$ ). C'est justement leur travail qui nous motive pour démontrer (3.1). 
Démonstration du théor ème 3.1. Soit $1<q<2$. Il est bien connu que $L_{a}(M, \tau)=\left(L_{1}(M, \tau), L_{2}(M, \tau)\right)_{0} \quad$ (avec des normes égales), oú $\theta$ est déterminé par la relation $1 / q=1-0 / 2: L_{q}(M, \tau)$ est donc $\theta$-hilbertien au sens de [P1]. D'autre part, $L_{q}(M, \tau)$ est 2-uniformément PL-convexe (cf. [F]). Une observation de G. Pisier montre que le module de la convexité uniforme réelle d'un espace $\theta$-hilbertien est équivalent à celui de la PL-convexité uniforme. En effet, cela résulte immédiatement du fait élémentaire suivant (qui est démontré par interpolation): Si $X$ est un espace de Banach 0-hilbertien, il existe alors une constante $\alpha=\alpha(\theta)$ telle que pour tous $x, y \in X$, on ait

$$
\left(\int_{0}^{2 \pi}\left\|x+\alpha e^{i t} y\right\|^{2} \frac{d t}{2 \pi}\right)^{1 / 2} \leqslant\left(\frac{\|x+y\|^{2}+\|x-y\|^{2}}{2}\right)^{1 / 2}
$$

(cf. [P2]; l'inégalité inverse est toujours vraie avec $\alpha=\pi / 2$ dans tous les espaces de Banach). En particulier, $L_{q}(M, \tau)$ est 2-uniformément convexe réel pour $1<q<2$. Par conséquent, il existe une constante $\alpha_{q}>0$ telle que toute fonction $f \in H_{q}\left(L_{q}(M, \tau)\right)$ vérifie

$$
\text { (3.2) } \quad\left(\|\hat{f}(0)\|_{\hat{L}_{q}(M, \tau)}^{2}+\alpha_{q}\|f-\hat{f}(0)\|_{H_{q}\left(L_{q}(M, \tau)\right)}^{2}\right)^{1 / 2} \leqslant\|f\|_{H_{q}\left(L_{q}(M, \tau)\right)} \text {. }
$$

Comme $L_{2}(M, \tau)$ est hilbertien, (3.2) est donc aussi vrai pour $q=2$ avec $\alpha_{2}=1$.

Soit maintenant $0<p \leqslant 1$. Soit $n=n(p)$ le plus petit entier $n$ tel que $1<n p \leqslant 2$. Posons $q=n p$. Alors $L_{q}(M, \tau)$ vérifie (3.2). On va démontrer que $L_{p}(M, \tau)$ vérifie $(3.1)$ avec $\alpha_{p}=n^{-(2 .-p) / p} \alpha_{q}$.

Rappelons la convention que la quasi-norme de $H_{p}\left(L_{q}(M, \tau)\right)$ est notée par $\|\cdot\|_{p q}$ ou par $\|\cdot\|_{p}$ si $p=q$. Prenons $f \in A F(M)$. Comme $1 / p=1 / q+\ldots+1 / q$ ( $n$ fois), d'après le théorème 2.1 , on trouve, pour $\varepsilon>0$ arbitrairement fixé, $n$ fonctions $f_{k}(1 \leqslant k \leqslant n)$ telles que

$$
f=\prod_{k=1}^{n} f_{k}, \quad \prod_{k=1}^{n}\left\|f_{k}\right\|_{q} \leqslant\|f\|_{p}+\varepsilon .
$$

Posons, pour $1 \leqslant k \leqslant n$,

$$
c_{k}=\prod_{j=1}^{k-1}\left\|\hat{f}_{j}(0)\right\|_{q}^{2}\left\|f_{k}-\hat{f}_{k}(0)\right\|_{q}^{2} \prod_{j=k+1}^{n}\left\|f_{j}\right\|_{q}^{2} .
$$

Alors

$$
\begin{aligned}
\|f-\hat{f}(0)\|^{2} & =\left\|\sum_{k=1}^{n} \prod_{j=1}^{k-1} \hat{f}_{j}(0)\left(f_{k}-\hat{f}_{j}(0)\right) \prod_{j=k+1}^{n} f_{j}\right\|_{p}^{2} \\
& \leqslant\left(\sum_{k=1}^{n} \prod_{j=1}^{k-1}\left\|\hat{f}_{j}(0)\right\|_{q}^{p}\left\|f_{k}-\hat{f}_{k}(0)\right\|_{q}^{p} \prod_{j=k+1}^{n}\left\|f_{j}\right\|_{q}^{p}\right)^{2 / p} \\
& \leqslant n^{(2-p) / p} \sum_{k=1}^{n} c_{k}
\end{aligned}
$$

Posons $\alpha_{p}=n^{-(2-p) / p} \alpha_{q}$. On déduit de (3.2) et (3.3) que

$$
\begin{aligned}
\|\hat{f}(0)\|_{p}^{2}+\alpha_{p}\|f-\hat{f}(0)\|_{p}^{2} & \leqslant \prod_{k=1}^{n}\left\|\hat{f}_{k}(0)\right\|_{q}^{2}+\alpha_{q} \sum_{k=1}^{n} c_{k} \\
& =\alpha_{q} \sum_{k=1}^{n-1} c_{k}+\left(\left\|\hat{f}_{n}(0)\right\|_{q}^{2}+\alpha_{q}\left\|f_{n}-\hat{f}_{n}(0)\right\|_{q}^{2}\right) \prod_{k=1}^{n-1}\left\|\hat{f}_{k}(0)\right\|_{q}^{2} \\
& \leqslant \alpha_{q} \sum_{k=1}^{n-1} c_{k}+\left\|f_{n}\right\|_{q}^{2} \prod_{k=1}^{n-1}\left\|\hat{f}_{k}(0)\right\|_{q}^{2} \\
& \leqslant \alpha_{q} \sum_{k=1}^{n-2} c_{k}+\left\|f_{n-1}\right\|_{q}^{2}\left\|f_{n}\right\|_{q}^{2} \prod_{k=1}^{n-2}\left\|\hat{f}_{k}(0)\right\|_{q}^{2} \\
& \leqslant \ldots \leqslant \prod_{k=1}^{n}\left\|f_{k}\right\|_{q}^{2} \leqslant\left(\|f\|_{p}+\varepsilon\right)^{2}
\end{aligned}
$$

En faisant $\varepsilon \rightarrow 0$, on obtient (3.1) si $f \in A F(M)$. La densité de $A F(M)$ dans $H_{p}\left(L_{p}(M, \tau)\right)$ montre alors $(3.1)$ pour toute $f$ dans $H_{p}\left(L_{p}(M, \tau)\right)$. Ceci termine la démonstration du théorème.

Remarque. La démonstration ci-dessus montre en mêrne temps que (3.1) est aussi vrai pour $p=1$ (avec $\alpha_{1}=1 / 2$ ). C'est un cas particulier du théorème de Haagerup et Pisier cité précédemment. D'autre part, leur théorème résulte aussi de (3.1) pour $p=1$. En effet, le théorème 3.1 est vrai pour tout espace non-commutatif $L_{p}(0<p \leqslant 1)$ associé à une algèbre de von Neumann arbitraire $M$ (pas nécessairement semi-finie) au sens de [H1]. On en expliquera la raison.

Soit $M$ une algèbre de von Neumann arbitraire. On note par $L_{p}(M)$ $(0<p \leqslant \infty)$ l'espace non-commutatif de Haagerup associé à $M$ au sens de [H1]. Il est connu que $L_{\infty}(M)=M$ et que $L_{1}(M)$ est isométrique à $M_{*}$, le prédual de $M$. Soit $0<p<\infty$. Le théorème suivant a été démontré dans [H2].

THÉRÈme 3.2. Soit $M$ une algebre de von Neumann arbitraire. Il existe un espace de Banach $X$ (un espace de $p$-Banach si $0<p<1$ ), une suite $\left(\left(M_{n}, \tau_{n}\right)\right)_{n} \geqslant 1$ d'algèbres de von Neumann finies avec des traces normales finies et fidèles $\tau_{n}$, une suite $\left(j_{n}\right)_{n \geqslant 1}$ d'injections isométriques avec $j_{n} ; L_{p}\left(M_{n}, \tau_{n}\right) \rightarrow X$ telles que

(i) $\bigcup_{n \geqslant 1} j_{n}\left(L_{p}\left(M_{n}, \tau_{n}\right)\right)$ soit dense dans $X$;

(ii) $j_{n}\left(L_{p}\left(M_{n}, \tau_{n}\right)\right) \subset j_{n+1}\left(L_{p}\left(M_{n+1}, \tau_{n+1}\right)\right), \forall n \geqslant 1$;

(iii) $L_{p}(M)$ soit isométrique à un sous-espace complémenté de $X$.

Soit $0<p \leqslant 1$. Comme la constante $\alpha$ dans (3.1) ne dépend que de $p$, en utilisant (i), (ii) ci-dessus et (3.1), on obtient pour tout $f \in H_{p}(X)$

$$
\left(\|\hat{f}(0)\|_{X}^{2}+\alpha_{p}\|f-\hat{f}(0)\|_{H_{p}(X)}^{2}\right)^{1 / 2} \leqslant\|f\|_{H_{p}(X)} .
$$

Par conséquent, (iii) du théorème 3.2 montre la même inégalité dans $H_{p}\left(L_{p}(M)\right)$. On démontre ainsi le théorème suivant: 
THÉORÈME 3.3. Soit $M$ une algèbre de von Neumann arbitraire (pas nécessairement semi-finie). Soit $0<p<1, L_{p}(M)$ est l'espace non-commutatif dt Haagerup associé à $M$. Alors toute fonction $f \in H_{p}\left(L_{p}(M)\right)$ vérifie

(3.5) $\quad\left(\|\hat{f}(0)\|_{L_{p}(M)}^{2}+\alpha_{p}\|f-\hat{f}(0)\|_{H_{p}\left(L_{p}(M, \tau)\right)}^{2}\right)^{1 / 2} \leqslant\|f\|_{H_{p}\left(L_{p}(M, \tau)\right)}$.

où $\alpha_{p}$ est la constante dans (3.1).

L'inégalité (3.1) est bien sûr un cas particulier de (3.5) puisque $L_{p}(M)$ ș réduit à $L_{v}(M, \tau)$ si $M$ est semi-finie munie d'une trace normale semi-finie e' fidèle $\tau$. (3.5) implique évidemment que $L_{p}(M)(0<p<1)$ est 2-uniformémen PL-convexe. Cela généralise le résultat de $[F]$ à tous les indices positifs.

Le reste de ce paragraphe est consacré à une étude brève de l'inégalité du type (3.4) pour un espace quasi-normé $X$. On a introduit dans [X] unt convexité uniforme complexe correspondant aux inégalités du type (3.4), i.e. l: $H$-convexité uniforme. Cette convexité uniforme complexe implique $l_{i}$ PL-convexité uniforme de [DGT]; elle est cependant impliquée par li PSH-convexité uniforme de Edgar [E1]. On ne sait pas si l'inverse de chaqu implication est vraie.

Soit $X$ un espace quasi-normé. Soit $0<p<\infty$. On définit, pour $\varepsilon>C$

$$
h_{p}^{X}(\varepsilon)=\inf \left\{\|f\|_{H_{p}(X)}-1:\|\hat{f}(0)\|=1,\|f-\hat{f}(0)\|_{H_{p}(X)} \geqslant \varepsilon, f \in H_{p}(X)\right\} .
$$

On dit que $X$ est uniformément $H_{p}$-convexe si $h_{p}^{X}(\varepsilon)>0$ pour tout $\varepsilon>0$ (cf. [X] Le théorème 3.3 montre que $L_{p}(M)(0<p \leqslant 1)$ est uniformément $H_{p}$-convex d'un module de convexité équivalente à $\varepsilon^{2}$ (le cas le meilleur possible).

On a caractérisé dans [X] les espaces uniformément $H_{p}$-convexifiables pa des inégalités pour des martingales de Hardy à valeurs dans ces espaces. $\mathrm{O}$ démontre, par cette caractérisation, le théorème de renormage. Le théorèm principal dans $[\mathrm{X}]$ est le suivant. On envoie le lecteur pour les notion intervenant dans ce théorème à $[\mathrm{X}]$ (et aussi à [G] pour la définition $\mathrm{d}$ martingales de Hardy).

THÉorème 3.4. Soit $X$ un espace quasi-normé. Soit $0<p<\infty$. Les assertion suivantes sont équivalentes.

(i) $X$ est uniformément $H_{p}$-convexifiable.

(ii) $X$ n'admet pas de $H_{p}$-arbres finis.

(iii) Il existe deux constantes $C>0,2 \leqslant q<\infty$ telles que toute martingal de Hardy $f=\left(f_{n}\right)_{n \geqslant 0}$ à valeurs dans $X$ vérifie

$$
\left(\sum_{n=0}^{\infty}\left\|d f_{n}\right\|_{p}^{q}\right)^{1 / q} \leqslant C\|f\|_{p}
$$

(iv) Il existe une quasi-norme équivalente $|\cdot|$ sur $X$, une constante $C^{\prime}>0$, $2 \leqslant r<\infty$ tels que

$$
h_{p}^{(X,|\cdot|)}(\varepsilon) \geqslant C^{\prime} \varepsilon^{r}
$$

Rappelons que $X$ a ARNP si toute fonction $f \in H_{\infty}^{D}(X)$ admet des limites radiales dans $X$ p.p. (cf. [Dow], [E2]), Il est connu que $X$ a ARNP si et seulement si pour un $0<p \leqslant \infty$ (et donc pour tout $0<p \leqslant \infty$ ), toute martingale de Hardy $L_{p}$-bornée à valeurs dans $X$ converge p.s. dans $X$ (cf. e.g. [GM]).

Remarques. (1) Soit $X$ un espace quasi-normé vérifiant (3.6); alors le complété $\tilde{X}$ de $X$ a ARNP. En effet, par un argument de transfert, on voit facilement qu'un bloc d'une martingale de Hardy est encore "essentiellement" une martingale de Hardy. Il s'ensuit que (3.6) est aussi valable pour les blocs des martingales de Hardy à valeurs dans $\not{X}$. Donc toute martingale de Hardy $L_{p}$-bornée à valeurs dans $\tilde{X}$ converge p.s. dans $\tilde{X}$. Par conséquent, $\tilde{X}$ a ARNP (en effet, $\tilde{X}$ a super ARNP puisque (3.6) est une super propriété).

(2) Les énoncés (ii) et (iii) du théorème 3.4 dans $[X]$ sont un peu différents de ceux du théorème ci-dessus. Dans les énoncés là-bas, il y a une condition de plus, i.e. " $X$ admet une quasi-norme équivalente qui est plurisousharmonique". Cette condition est en fait superflue. En supposant (ii), (iii) ci ${ }^{-d e s s u s, ~} X$ admet automatiquement une quasi-norme équivalente plurisousharmonique. En effet, on a démontré dans [X] que (ii) implique (iii) sans aucune condition supplémentaire. Par la remarque précédente, (iii) implique que le complété de $X$ a ARNP. Un résultat de Kalton [K] montre alors que $X$ admet une quasi-norme équivalente plurisousharmonique.

Par le théorème 3.3 et la remarque (1) ci-dessus, on obtient le corollaire suivant:

Corollatre 3.5. Soit $M$ une algèbre de von Neumann arbitraire. Soit $0<p<1$. Alors l'espace non-commutatif $L_{p}(M)$ de Haagerup associé à $M$ a super ARNP. En particulier, $L_{p}(M, \tau)$ a super ARNP si $(M, \tau)$ est une algèbre de von Neumann semi-finie munie d'une trace semi-finie normale et fidèle $\tau$.

A l'aide du corollaire 3.5 , on vérifie facilement que le théorème 2.1 est encore vrai si on remplace tous les $H_{p}\left(L_{u}(M, \tau)\right)$ par $H_{p}^{D}\left(L_{p}(M, \tau)\right)$ puisque le seul problème est d'approcher les fonctions dans $H_{p}^{D}\left(L_{q}(M, \tau)\right)$ par des polynômes (voir la démonstration du théorème 2.1 , surtout la dernière étape) On peut maintenant le résoudre. En effet, on a un résultat général simple.

Proposition 3.6. Soit $X$ un espace de quasi-Banach avec ARNP. Soit $0<p<\infty$. Alors toute fonction dans $H_{p}^{D}(X)$ est la limite d'une suite de polynômes analytiques à valeurs dans $X$. Par conséquent, en identifiant les fonctions dans $H_{p}^{D}(X)$ avec leurs limites radiales, $H_{p}^{D}(X)$ coincide avec $H_{p}(X)$.

Démonstration. Il est connu que $X$ a ARNP si et seulement si pour un $0<p \leqslant \infty$ (et donc pour tout $0<p \leqslant \infty$ ) toute fonction dans $H_{p}^{D}(X)$ admet les limites radiales dans $X$ presque partout (cf. [E2], [Dow], [GM]).

Soit maintenant $X$ un espace de quasi-Banach avec ARNP. Par le théorème de Kalton cité ci-dessus, $X$ admet une quasi-norme équivalente plurisoushar- 
monique. Supposons donc que la quasi-norme de $X$ elle-même est plurisous harmonique. Si $f$ est une fonction analytique sur le disque unité $D$ à valeur dans $X, \log \|f(z)\|$ est sousharmonique sur $D$. On en déduit que pour tou $0<p<\infty$, il existe une constante $C=C(p)$ telle que toute fonction $f \in H_{p}^{D}(X$ vérifie

$$
\left.\left(\int \sup _{0<r<1} \| f\left(r e^{i t}\right)\right\} \|^{p} \frac{d t}{2 \pi}\right)^{1 / p} \leqslant C\|f\|_{H p(X)}
$$

Fixons $0<p<\infty$. Soit $f \in H_{p}^{D}(X)$. Posons

$$
f\left(e^{i t}\right)=\lim _{r \rightarrow 1} f\left(r e^{i t}\right), \quad t \in[0,2 \pi] .
$$

(3.7) montre que $f \in L_{p}(X, d t)$ et que

$$
\|f\|_{L_{p}(X, d t)} \leqslant C\|f\|_{H_{p}^{D}(X)} .
$$
On a

L'inverse de (3.8) est toujours vrai puisque $\|\cdot\|^{p}$ est sousharmonique sur $L$

$$
\|f\|_{H_{p}^{D}(X)} \leqslant\|f\|_{L_{p}(X, d t)} .
$$

Posons $f_{r}(z)=f(r z) \quad(0<r<1, z \in D)$. Alors $f_{r}(z)$ peut être uniformémer approché par des polynômes à valeurs dans $X$ sur le disque unité fermé $I$ Pour montrer que $f$ peut être approché dans $H_{p}^{D}(X)$ par des polynômes, il suff donc de montrer que $f_{r}$ converge vers $f$ dans $H_{p}^{D}(X)$. Or cette dernière assertio est une consequence immédiate du (3.7). En effet, comme $\lim _{r \rightarrow 1} f\left(r e^{i t}\right)=f\left(e^{i}\right.$ p.p. et comme

$$
\left\|f\left(r e^{i t}\right)\right\| \leqslant \sup _{0<r<1}\left\|f\left(r e^{i t}\right)\right\| \in L_{p} \quad(0<r \leqslant 1),
$$

d'après le théorème de convergence dominée des intégrales, on a

$$
\begin{aligned}
\lim _{r \rightarrow 1}\left\|f-f_{r}\right\|_{r_{p}^{p}(x)}^{p} & =\lim _{r \rightarrow 1} \sup _{0<r^{\prime}<1} \int\left\|f\left(r^{\prime} e^{i t}\right)-f\left(r^{\prime} r e^{i t}\right)\right\|^{p} \frac{d t}{2 \pi} \\
& \leqslant \lim _{r \rightarrow 1} \int\left\|f\left(e^{i t}\right)-f\left(r e^{i t}\right)\right\|^{p} \frac{d t}{2 \pi} \quad \text { (d'après (3.7)) } \\
& =0 .
\end{aligned}
$$

COROllatre 3.7. Soit $(M, \tau)$ une algèbre de von Neumann semi-finie. Soient $q, p_{i}, q_{i}$ comme dans le théorème $2.1(i=0,1)$. Alors pour toute fonctio $f \in H_{p}^{D}\left(L_{q}(M, \tau)\right) \cap A F(M)$ (en identifiant $f$ avec sa limite radiale) et tout $\varepsilon>0$, existe $g \in H_{p_{0}}^{D}\left(L_{q_{0}}(M, \tau)\right), h \in H_{p_{1}}^{D}\left(L_{q_{1}}(M, \tau)\right)$ telles que

$$
\begin{gathered}
f(z)=g(z) h(z), \quad \forall z \in D, \\
\|g\|_{H_{p_{0}}^{D}\left(L_{q 0}(M, \tau)\right)}\|h\|_{H_{p_{1}}^{D}\left(L_{q_{1}}(M, \tau)\right)} \leqslant\|f\|_{H_{p}^{D}\left(L_{\eta}(M, \tau)\right)}+\varepsilon,
\end{gathered}
$$

$\S 4$. Interpolation complexe. Soient $1 \leqslant q_{0}, q_{1} \leqslant \infty$. Soit $0<0<1$. Il est bien connu que

$$
\left(L_{q_{0}}(M, \tau), L_{q_{1}}(M, \tau)\right)_{0}=L_{q}(M, \tau) \quad \text { (normes égales), }
$$

où $1 / q=(1-0) / q_{0}+\theta / q_{1}$. Si $1<q<\infty$, la projection de Riesz est bornée dans $L_{p}\left(L_{q}(M, \tau)\right)$ pour tout $1<p<\infty$ (cf. [BGM]). On en déduit que si $1<p_{0}, p_{1}$, $q_{0}, q_{1}<\infty$ et si $0<0<1$, alors

$$
\left(H_{p_{0}}\left(L_{q 0}(M, \tau)\right), H_{p_{1}}\left(L_{q 1}(M, \tau)\right)\right)_{0}=H_{p}\left(L_{q}(M, \tau)\right)
$$

(normes équivalentes), où $1 / p=(1-0) / p_{0}+0 / p_{1}, 1 / q=(1-0) / q_{0}+0 / q_{1}$.

Dans ce paragraphe on va étendre (4.1), (4.2) à tous les indices en utilisant le théorème de factorisation 2.1. Comme $L_{q}(M, \tau)$ n'est pas un espace de Banach pour $0<q<1$, on va utiliser une petite modification de la méthode d'interpolation complexe classique de Calderón. On la rappelle brièvement (pour plus d'information, voir $[\mathrm{BL}],[\mathrm{CMS}]$ et $[\mathrm{Kr}]$ )

Notons par $S=\{z \in C: 0<\operatorname{Re} z<1\}$ la bande ouverte dans le plan complexe et $\widetilde{S}$ sa fermeture. Notons par $A(S)$ l'espace des fonctions à valeurs complexes, analytiques dans $S$, continues et bornées dans $S$. Soit $\left(X_{0}, X_{1}\right)$ un couple d'interpolation d'espaces de quasi-Banach. Posons

$$
\begin{aligned}
& \text { 雨 }\left(X_{0}, X_{1}\right)=\left\{f: f(z)=\sum_{k=1}^{m} f_{k}(z) x_{k}, x_{k} \in X_{0} \cap X_{1},\right. \\
& \left.f_{k} \in A(S), 1 \leqslant k \leqslant n, n \in N\right\} . \\
& \|f\|_{\mathscr{F}\left(X_{0}, X_{1}\right)}=\sup _{t \in \mathbb{R}}\left\{\left\{\|f(i t)\|_{X_{0}},\|f(1+i t)\|_{X_{1}}\right\} .\right.
\end{aligned}
$$

Soit $0<0<1$. La norme d'interpolation complexe d'indice $\theta$ sur $X_{0} \cap X_{1}$ est définie par

$$
\left.\|x\|_{\theta}=\inf \left\{\|f\|_{\mathscr{F}\left(X_{0}, X_{1}\right)}: f(\theta)=x, f \in X_{0}, X_{1}\right)\right\}, \quad x \in X_{0} \cap X_{1} .
$$

On note par $\left(X_{0}, X_{1}\right)_{0}$ le complété de $X_{0} \cap X_{1}$ par rapport à $\|\cdot\|_{\vartheta}$. Ul est connu que si $X_{0}, X_{1}$ sont des espaces de Banach, cette définition coïncide avec la classicue.

On étend maintenant $(4.1)$ à tous les indices dans $] 0, \infty]$.

THFOREME 4.1. Soient $0<0<1,0<q_{0}<q_{1} \leqslant \infty$. Alors

(4.3) $\quad\left(L_{q_{0}}(M, \tau), L_{q_{1}}(M, \tau)\right)_{0}=L_{q}(M, \tau) \quad$ (normes égales)

oì $1 / q=(1-0) / q_{0}+0 / q_{1}$.

Démonstration. Elle se divise en plusieurs étapes.

(i) On a l'inclusion de norme $\leqslant 1$ :

$$
L_{q^{\prime}}(M, \tau) \subset\left(L_{q_{0}}(M, \tau), L_{q_{1}}(M, \tau)\right)_{0} .
$$


Il suffit de démontrer que pour tout $a \in F(M)$ on a $\|a\|_{\theta} \leqslant\|a\|_{q}$. Soit donc $a \in F(M)$. Soit $a=u|a|$ la décomposition polaire de $a$. Choisissons un entier $n$ positif tel que $n q_{0}>1$. Alors $a$ s'écrit comme suit:

$$
a=u|a|=\left.u \underbrace{|a|^{1 / n} \ldots \mid a}_{n \text { fois }}\right|^{1 / n}=a_{1} a_{2} \ldots a_{n},
$$

où $a_{1}=u|a|^{1 / n}, a_{k}=|a|^{1 / n}(2 \leqslant k \leqslant n)$. Donc $a_{k} \in L_{n q_{0}}(M, \tau)(1 \leqslant k \leqslant n)$. Soit $\varepsilon>0$. D'après (4.1) (appliqué à $L_{n q_{0}}(M, \tau), L_{n q_{1}}(M, \tau), L_{n q}(M, \tau)$ ), il existe $f_{k} \in \mathscr{F}\left(L_{n q_{0}}(M, \tau), L_{n q_{1}}(M, \tau)\right)(1 \leqslant k \leqslant n)$ tel que

$$
f_{k}(\theta)=a_{k}, \quad\left\|f_{k}\right\|_{\mathscr{F}\left(L_{n q 0}(M, \tau), L_{n q_{1}}(M, \tau)\right)} \leqslant\left\|a_{k}\right\|_{L_{n q}(M, \tau)}+\varepsilon
$$

$(1 \leqslant k \leqslant n)$. Posons $f=f_{1} \ldots f_{n}$. Il est évident que

$$
f(\theta)=a, \quad f \in \mathscr{F}\left(L_{q_{0}}(M, \tau), L_{q_{1}}(M, \tau)\right) \text {. }
$$

On a de plus, d'après l'inégalité de Hölder,

$$
\begin{gathered}
\|f(i t)\|_{q_{0}} \leqslant \prod_{k=1}^{n}\left\|f_{k}(i t)\right\|_{n q_{0}} \leqslant \prod_{k=1}^{n}\left(\left\|a_{k}\right\|_{n q}+\varepsilon\right), \\
\|f(1+i t)\|_{q_{1}} \leqslant \prod_{k=1}^{n}\left\|f_{k}(1+i t)\right\|_{n q_{1}} \leqslant \prod_{k=1}^{n}\left(\left\|a_{k}\right\|_{n q}+\varepsilon\right) .
\end{gathered}
$$

D'où

$$
\|f\|_{\mathscr{F}\left(L_{q_{0}}(M, \tau), L_{q_{1}}(M, \tau)\right)} \leqslant\|a\|_{q}+o(\varepsilon),
$$

ce qui donne (4.4) en faisant $\varepsilon \rightarrow 0$.

(ii) Supposons $\tau(1)=1$, On a

(4.5) $\quad\left(L_{q_{0}}(M, \tau), L_{q_{1}}(M, \tau)\right)_{\theta} \subset L_{q}(M, \tau) \quad$ (inclusion de norme $\left.\leqslant 1\right)$.

Il suffit de démontrer que tout opérateur $a \in F(M)$ vérifie

$$
\|a\|_{q} \leqslant\|a\|_{\theta} .
$$

Prenons $a \in F(M)$. Soit $f \in \mathscr{F}\left(L_{q_{0}}(M, \tau), L_{q_{1}}(M, \tau)\right)$ tel que $f(\theta)=a$. Par approximation, on peut supposer que $f$ est de la forme suivante:

$$
f=\sum_{k=1}^{n} f_{k} a_{k}, \quad f_{k} \in A(S), a_{k} \in F(M)
$$

On va démontrer que

$$
\|a\|_{q} \leqslant\|f\|_{\mathcal{F}\left(L_{q_{0}}(M, \tau), L_{q_{1}}(M, \tau)\right)} .
$$

Cela donne immédiatement $\left(4.5^{\prime}\right)$.

En multipliant $f$ par $e^{\delta(z-\theta)^{2}}(\delta>0$ arbitrairement fixé), on peut évidemment supposer que $f(z)$ tend vers zéro très vite dans $L_{q_{0}}(M, \tau) \cap L_{q_{1}}(M, \tau)=L_{q_{1}}(M, \tau)$ (en remarquant $\tau(1)=1$ ) quand $z$ tend vers linfini. On suppose donc que pour $0 \leqslant s \leqslant 1,0<p<\infty$,

$$
\int_{-\infty}^{\infty}\|f(s+i t)\|_{q_{1}}^{p} d t<\infty
$$

Choisissons $n \in N$ tel que $n q_{0}>1$. Soit $\varepsilon>0$. D'après le théorème de Riemann sur la transformation conforme et le corollaire 2.3 , il existe $n$ fonctions $g_{1}, \ldots, g_{n}$ qui sont analytiques et bornées sur $S$ à valeurs dans $M$ et qui vérifient

$$
\left\{\begin{array}{l}
f(z)=\prod_{k=n}^{n} g_{k}(z), \quad \forall z \in \bar{S}, \\
\left\|g_{k}(z)\right\|_{n r} \leqslant\|f(z)\|_{r}^{1 / n}+\varepsilon, \quad \forall z \in S \backslash S \text { et } 0<r<\infty
\end{array}\right.
$$

(pour appliquer le corollaire 2.3 et aussi la remarque 2.4 on transforme d'abord $f$ par une transformation conforme en une fonction analytique sur le disque unité, puis on utilise le corollaire 2.3 pour cette dernière fonction et on revient finalement sur la bande). $g_{k}$ est analytique et bornée sur $S$ à valeurs dans $L_{n q_{0}}(M, \tau)=L_{n q_{0}}(M, \tau)+L_{n q_{1}}(M, \tau)$. Posons $b_{k}=g_{k}(\theta) \quad(1 \leqslant k \leqslant n)$. D'après $(4.8), a=\prod_{k=1}^{n} b_{k}$.

Soit $\left(\chi_{m}\right)_{m \geqslant 1}$ une suite de fonctions continues positives sur $\boldsymbol{R}$ vérifiant

$$
\int_{-\infty}^{\infty} \chi_{m}(t) d t=1 \quad(m \geqslant 1)
$$

Posons

$$
\chi_{m}(t)=0 \quad \text { si }|t|>1 / m(m \geqslant 1) \text {. }
$$

$$
G_{k m}(z):=\int_{-\infty}^{\infty} g_{k}(z+i t) \chi_{m}(t) d t \quad(1 \leqslant k \leqslant n, m \geqslant 1) .
$$

Alors $G_{k m}$ est analytique et borné sur $S(1 \leqslant k \leqslant n, m \geqslant 1)$. De plus, (4.7), (4.8) montrent

$$
\int_{-\infty}^{\infty}\left\|G_{k m}(j+i t)\right\|_{n q_{l}} d t<\infty \quad(1 \leqslant k \leqslant n, m \geqslant 1, j, l=0,1) .
$$

On en déduit alors que $G_{k m}$, comme une fonction à valeurs dans $L_{n q_{0}}(M, \tau)$ ou dans $L_{n q_{1}}(M, \tau)$, est continue sur $\bar{S} \backslash S(1 \leqslant k \leqslant n, m \geqslant 1)$. D'où $G_{k m}$ est continue sur $S$ puisque $G_{\mathrm{km}}$ se présente dans la bande comme intégrale de Poisson de sa limite au bord. Comme $n q_{1}>n q_{0}>1$, on utilise (4.1) pour $G_{k m}$ et on obtient

Mais (4.8) implique

de même,

$$
\left\|G_{k m}(0)\right\|_{n q} \leqslant\left\|G_{k m H}\right\|_{; r}\left(L_{\left.n q_{0}(M, z), L_{n \mu_{1}}(M, \tau)\right)} .\right.
$$

$$
\begin{aligned}
\left\|G_{k m}\left(i t^{\prime}\right)\right\|_{n q_{0}} & \leqslant \int_{-\infty}^{\infty}\left\|g_{k}\left(i t^{\prime}+i t\right)\right\|_{n q_{0}} \chi_{m}(t) d t \\
& \leqslant \sup _{t \in \mathbb{R}}\|f(i t)\|_{q_{0}}^{1 / n}+\varepsilon \quad \forall t^{\prime} \in \boldsymbol{R} ;
\end{aligned}
$$

$$
\left\|G_{k m}\left(1+i t^{\prime}\right)\right\|_{m q_{1}} \leqslant \sup \|f(1+i t)\|_{q_{1}}^{1 / n}+\varepsilon, \quad \forall t^{\prime} \in \boldsymbol{R} .
$$


D'où

$(4.10)^{*} \quad\left\|G_{k m}\right\|_{\mathscr{F}\left(L_{n q 0}(M, \tau), L_{n q 1}(M, \tau)\right)} \leqslant\|f\|_{\mathscr{F}\left(L_{q 0}(M, \tau), L_{q_{1}}(M, \tau)\right\}}+\delta$.

(4.9) et $(4.10)$ nous donnent

$$
\left\|G_{k m}(0)\right\|_{n q} \leqslant\|f\|_{\left.i / L_{q 0}(M, \tau), L_{q_{1}}(M, \tau)\right)}+\varepsilon \quad(1 \leqslant k \leqslant n, m \geqslant 1) .
$$

Faisant $m \rightarrow \infty$, on obtient

$$
\left\|g_{k}(\theta)\right\|_{n q} \leqslant\|f\|_{\mathscr{S}^{\prime}\left(J_{q_{0}}(M, \tau), L_{q_{1}}(M, \tau)\right)}^{1 / n}+\varepsilon \quad(1 \leqslant k \leqslant n) .
$$

On en déduit, par l'inégalité de Hölder, que

$$
\|a\|_{q} \leqslant \prod_{k=1}^{n}\left\|g_{k}(\theta)\right\|_{m q} \leqslant\left(\|f\|_{\mathscr{F}\left(L_{q 0}(M, \tau), L_{q_{1}}(M, \tau)\right)}+\ell\right)^{n} .
$$

En faisant $\varepsilon \rightarrow 0$, on obtient (4.6). Cela démontre (ii).

(iii) Cas général:

$$
\left(L_{q_{0}}(M, \tau), L_{q_{1}}(M, \tau)\right)_{\theta} \subset L_{q}(M, \tau) .
$$

Soit $a \in F(M)$. Il suffit de démontrer que $\|a\|_{q} \leqslant\|a\|_{\theta}$. Prenons une projection $e \in M$ telle que $\tau(e)<\infty$ et $e a e=a$. Définissons

$$
P_{e}: B(H) \rightarrow B(H), \quad b \mapsto e b e .
$$

Alors $P_{e}$ est une projection de norme 1 dans $L_{r}(M, \tau)(0<r<\infty) . P_{u} L_{r}(M, \tau)$ s'identifie évidemment à $L_{r}\left(M_{e}, \tau_{e}\right)$. D'après (ii), on a

D'où

$$
\left(L_{q 0}\left(M_{e}, \tau_{e}\right), L_{q_{1}}\left(M_{e}, \tau_{e}\right)\right)_{e} \subset L_{q}\left(M_{e}, \tau_{e}\right) .
$$

$$
\|a\|_{q} \leqslant\left\|a_{e}\right\|_{\left(L_{q_{0}}\left(M_{e}, \tau_{e}\right), L_{q_{1}}\left(M_{e}, \tau_{e}\right)\right)_{0}} .
$$

Il s'ensuit, par interpolation, que

$$
\|a\|_{q} \leqslant\|a\|_{\left(L_{q_{0}}(M, \tau), L_{q_{1}}(M, \tau)\right)_{0}} .
$$

Cela démontre (iii). On achève donc la démonstration du théorème.

Notons que (4.3) est connu pour l'interpolation réelle (cf. [PS])

Pour l'extension de (4.2) à tous les indices, on a encore besoin de la proposition suivante:

Proposition 4.2. Soit $(\Omega, \mu)$ un espace mestré arbitraire. Soit $\left(X_{0}, X_{1}\right)$ ur. couple d'interpolation d'espaces de quasi-Banach. On a, pour $0<p_{0}, p_{1}<\infty$ $0<0<1$,

$$
\left(L_{p_{0}}\left(X_{0}\right), L_{p_{1}}\left(X_{1}\right)\right)_{\theta}=L_{p}\left(\left(X_{0}, X_{1}\right)_{0}\right)
$$

où $1 / p=(1-0) / p_{0}+\theta / p_{1}$.

Démonstration. Il suffit de démontrer que pour toute fonction $f$ à va. leurs dans $X_{0} \cap X_{1}$, on a

$$
\|f\|_{\left(X_{p, j}\left(X_{i}\right), L_{p 1}\left(X_{1}\right)_{e}\right.}=\|f\|_{L_{p}\left(\left(X_{0}, X_{1}\right)_{\theta}\right)}
$$

La démonstration de l'inégalité

$$
\|f\|_{\left(x_{p_{0}}\left(X_{0}\right), L_{p_{1}}\left(X_{1}\right)_{\theta}\right.} \leqslant\|f\|_{L_{p}\left(\left(X_{0}, X_{1}\right)_{\theta}\right)}
$$

est identique au cas des espaces de Banach (cf. [BL], p. 108).

Pour l'inverse, on prend une fonction $F \in \mathscr{F}\left(L_{p_{0}}\left(X_{0}\right), L_{p_{1}}\left(X_{1}\right)\right)$ telle que $F(\theta)=f$. D'après le théorème 1 de [CMS] (cf. aussi le lemme 4.3.2 dans [BL]), pour tout $(\alpha) \Omega$, on a

$$
\begin{aligned}
\log \left\|f^{\prime}(\omega)\right\|_{\theta} \leqslant & \int_{\infty=\infty}^{\infty} \log \| F\left(i t,(\omega) \|_{X_{0}} P_{0}(\theta, t) d t\right. \\
& +\int_{-\infty}^{\infty} \log \|F(1+i t, \omega)\|_{X_{1}} P_{1}(0, t) d t,
\end{aligned}
$$

où $P_{j}(0, t)(j=0,1)$ sont les noyaux de Poisson pour la bande $S$. On en déduit que

$$
\begin{aligned}
\|f\| \lambda_{p}\left(\left(X_{n_{2}} X_{1}\right)_{\theta}\right) \leqslant & \int\left[\exp \left(p \int_{-\infty}^{\infty} \log \|F(i t, \omega)\|_{x_{0}} P_{0}(0, t) d t\right)\right. \\
& \left.\quad \times \exp \left(p \int_{-\infty}^{\infty} \log \|F(1+i t, \omega)\|_{X_{1}} P_{1}(0, t) d t\right)\right] d \mu(\omega) .
\end{aligned}
$$

En appliquant les inégalités de Hölder et de Jensen, on obtient

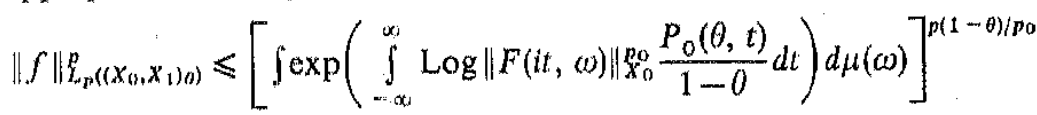

$$
\begin{aligned}
& \times\left[\int \exp \left(\int_{-\infty}^{\infty} \log \|F(1+i t, \omega)\| p_{1} \frac{P_{1}(0, t)}{0} d t\right) d \mu(\omega)\right]^{p \theta / p}
\end{aligned}
$$

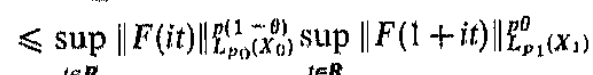

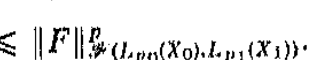

Il s'ensuit donc

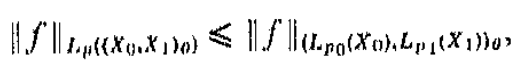

ce qui démontre la proposition.

Theorima: 4.3. Solent $0<p_{0}, p_{1}, q_{0}, q_{1}<\infty$. Soit $0<0<1$. Alors

$$
\left(H_{p_{0}}\left(L_{q_{0}}(M, \tau)\right), H_{p_{1}}\left(L_{i, 1}(M, \tau)\right)\right)_{0}=H_{p}\left(L_{q}(M, \tau)\right)
$$

(quasi-normes équivalentes), où $1 / p=(1-0) / p_{0}+0 / p_{1}, 1 / q=(1-0) / q_{0}+0 / q_{1}$.

Démonstration. L'inclusion

$$
\left(H_{p_{0}}\left(L_{q 0}(M, \tau)\right), H_{p_{1}}\left(L_{q_{1}}(M, \tau)\right)\right)_{0} \subset H_{p}\left(L_{q}(M, \tau)\right)
$$

résulte de la proposition 4.2 et du théorème 4.1. On démontre l'inclusion inverse similairement à la démonstration de l'inclusion $L_{4}(M, \tau) \subset$ 
$\left(L_{q_{0}}(M, \tau), L_{q_{1}}(M, \tau)\right)_{\theta}$ dans la démonstration du théorème 4.1, en utilisant (4.2) et le théorème 2.1. En effet, soit $f \in A F(M)$. Choisissons $n \in N$ tel que $n p_{j}$, $n q_{j}>1(j=0,1)$. D'après le théorème 2.1 , on trouve des fonctions $f_{k} \in A F(M)$ $(1 \leqslant k \leqslant n)$ telles que

$$
f=\prod_{k=1}^{n} f_{k}, \quad\|f\|_{p q} \leqslant \prod_{k=1}^{n}\left\|f_{k}\right\|_{n p, n q}+\varepsilon
$$

où $\varepsilon$ est un nombre positif donné. On a évidemment

$$
f_{k} \in H_{n p_{0}}\left(L_{n q_{0}}(M, \tau)\right) \cap H_{n p_{1}}\left(L_{n q_{1}}(M, \tau)\right) \quad(1 \leqslant k \leqslant n) .
$$

En utilisant (4.2), on achève la démonstration comme dans celle du théorème 4.1(i). .

Remarques. (i) L'idée essentielle de la démonstration ci-dessus est l'utilisation du théorème de factorisation pour ramener des indices inférieurs à 1 à ceux supérieurs à 1 et donc pour utiliser la "UMD propriété" des espaces correspondants. Cette idée a été déjà utilisée dans $[\mathrm{BP}]$ (cf. la démonstration du théorème 2.2 de [BP]). Remarquons que ce raisonnement n'est plus valable si on laisse certains des indices $p_{i}, q_{i}(i=0,1)$ dans (4.11) prendre l'infini comme valeur. On ne sait pas si (4.11) est encore vrai dans ce dernier cas. La réponse est probablement positive.

(ii) (4.11), combiné avec le théorème 4.7.2 de [BL], donne quelques résultats similaires pour l'interpolation réelle. Par example, si $0<p_{0}, p_{1}<\infty$ et $0<\theta<1$, alors

(4.12) $\quad\left(H_{p_{0}}\left(L_{p_{0}}(M, \tau)\right), H_{p_{1}}\left(L_{p_{1}}(M, \tau)\right)\right)_{\theta p}=H_{p}\left(L_{p}(M, \tau)\right)$,

où $1 / p=(1-\theta) / p_{0}+\theta / p_{1} ;$ si $1<p_{1}, q_{1}<\infty$ et si $p_{i}, q_{i}(i=0,1), p, q$ vérifient la condition du théorème 4.3 , alors

$$
\left(H_{p_{0}}\left(L_{q_{0}}(M, \tau)\right), H_{p_{1}}\left(L_{q_{1}}(M, \tau)\right)\right)_{\theta_{p}}=H_{p}\left(L_{q p}(M, \tau)\right),
$$

où $L_{q p}(M, \tau)$ est l'espace non-commutatif de Lorentz défini par

$$
L_{q p}(M, \tau)=\left\{a: a \text { mesurable et } \mu(a) \in L_{q p}(0, \infty)\right\},
$$

muni de la norme

$$
\|a\|_{q p}=\left(\int_{0}^{\infty}\left(t^{1 / q} \mu_{t}(a)\right)^{p} \frac{d t}{t}\right)^{1 / p} .
$$

(4.12), (4.13) résultent de (4.11), du théorème 4.7.2 de [BL] et du fait élémentaire suivant: si $1<p_{0}, q_{0}, p_{1}, q_{1}<\infty$, alors

$$
\left(H_{p_{0}}\left(L_{q 0}(M, \tau)\right), H_{p_{1}}\left(L_{q_{1}}(M, \tau)\right)\right)_{\theta p}=H_{p}\left(L_{q p}(M, \tau)\right),
$$

où $1 / p=(1-\theta) / p_{0}+\theta / p_{1}, 1 / q=(1-\theta) / q_{0}+\theta / q_{1}$. On laisse les détails au lecteur.

\section{Références}

[BL] J. Bergh and J. Lörström, Interpolation Spaces, Springer, Berlin 1976

[BGM] E. Berkson, T. A. Gillespie and P. S. Muhly, Abstract spectral decompositions guaranteed by the Hilbert transform, Proc. London Math. Soc. 53 (1986), 489-517.

[BP] O. Blasco and A. Pełczy n'ski, Theorems of Hardy and Paley for vector valued analytic functions and related classess of Banach spaces, Trans. Amer. Math. Soc., à paraître.

[CMS] M. Cwikel, M. Milman and Y. Sagher, Complex interpolation of some quasi-Banach spaces, J. Funct. Anal. 65 (1986), 339 347.

[DGT] W. J. Davis, D. J, H. Garling and N. Tomczak-Jaegermann, The complex convexity of quasi-normed spaces, ibid. 55 (1984), 110 150.

[D] A. Devinatz, The factorization of operator valued functions, Ann. of Math. 73 (1961), 458:495.

[Dow] P. M. Dowling, Representable operators and the analytic Radon-Nikodym property in Banach spaces, Proc. Royal Irish Acad. Sect. A 85 (1985), 143-150.

[E1] G. A. Edgar, Complex martingale convergence, in: Lecture Notes in Math. 1116, Springer $1985,38-59$.

[E2] -, Analytic martingale convergence, J. Funct. Anal. 69 (1986), 268-280.

[F] T. F a ck, Type and cotype inequalities for non commutative $L^{p}$-spaces, J. Operator Theory 17 (1987), 255 279 .

[FK] T. Fack and H. Kosaki, Generalized snumbers of $\tau$-measurable operators, Pacific J. Math. 123 (1986), 269-300.

[G] D. J. H. Garling, On murtingales with values in a complex Banach space, Math. Proc. Cambridge Philos. Soc. (1988), à paraître.

[GM] N. Ghoussoub and B. Maurey, Plurisubharmonic martingales and barriers in complex quasi-Banach spaces, preprint, 1988.

[H1] U. Haagerup, $L^{p}$-spaces associated with an arbitrary von Neumann algebra, in: Colloq Internat. CNRS 274 (1977), 175 184

[H2] -, Non-commutative integration theory, Lecture given at the Symposium in Pure Mathematics of the Amer. Math. Soc., Queens University, Kingston, Ontario, 1980.

[HP] U. Haagerup and G. Pisier, Factorization of analytic functions with values in non-commutative $L_{1}$-spaces and applications, preprint, 1988.

[K] N. J. Kalt on, Differentiability properties of vector-valued functions, in: Lecture Notes in Math. 1221, Springer, 1985, 141-181.

[Kr] P. K ree, Interpolation d'espaces qui ne sont ni normés ni complets. Applications, Ann. Inst. Fourier (Grenoble) 17 (1967), 137-174.

[N] E. Nelson, Notes on non-commutative integration, J. Funct. Anal. 15 (1974) 103116.

[O] V. I. Ovchinnikov, s-Numbers of measurable operators, Functional Anal. Appl. 4 (1970), 236242 .

[PS] J. Peetre and G. Sparr, Interpolation and non-commutative integration, Ann. Mat. Pura Appl. 104 (1975), $187 \% 207$.

[P1] G. Pisier, Some applications of the complex interpolation method to Banach lattices, J. Analyse Math. 35 (1979), 264-281.

[P2] -- Sur les espaces de Banach K-convexes, Sérm. d'Analyse Fonctionnelle 79/80, Ecole Polytechnique, Palaiscau, exp. no. 11.

[S] D. Sarason, Generalized interpolation in $H^{\infty}$, Trans. Amer. Math. Soc. 127 (1967) 179. 203.

7 - Studia Mathematica 95.3 
[Se] I. E. Segal, A non-commutative extension of abstract integration, Ann. of Math. 57 (1953) 401-457.

[X] Q. Xu, Inégalités pour les martingales de Hardy et renormage des espaces quasi-normés, $\mathrm{C} . \mathrm{R}$. Acad. Sci. Paris 306 (1988), 601-604.

UNIVERSITE DE WUHAN

ÉQUIPE D'ANALYSE

U. A. No. 754 au C.N.R.S.

UNIVERSITÉ PARIS VI

4. P1. Jussieu, 75252 Paris Cedex 05, France

\section{STUDIA MATHEMATICA}

\section{Contents of forthcoming issues}

\section{Volume 96, Number 1}

S. Broom, Sharp weights and BMO-preserving homeomorphisms.

-, An interpolation theorem with $A_{p}$-weighted $L^{p}$ spaces.

S. RoLiwicz, On projections on subspaces of codimension one.

A. PIETsch, Type and cotype numbers of operators on Banach spaces.

D. LI, Quantitative unconditionality of Banach spaces $E$ for which $\mathscr{K}(E)$ is an $M$-ideal in $\mathscr{L}(E)$.

S. Момм, Partial differential operators of infinite order with constant coefficients on the space of analytic functions on the polydisc.

A. PARUSINSKK, Gradient homotopies of gradient vector fields.

B. Bi:AUZAMY, An operator on al separable Hilbert space with all polynomials hypercyclic.

P. Pycir-TAizersKa, Approximation properties of the partial sums of Fourier series of some almost periodic functions.

\section{Volume 96, Number 2}

J. D. McPlyall, A weighted interpolation problem for analytic functions.

G. BLoWER, A multiplier characterization of analytic UMD spaces.

E. V. FirReyra, Weighted Lorentz norm inequalities for integral operators.

B. KAMIŃSKI, An axiomatic definition of the entropy of a $\mathbf{Z}^{d}$-action on a Lebesgue space.

E. SAWYER, Boundedness of classical operators on classical Lorentz spaces.

W. Żel $\wedge$ ZKKO, A density theorem for $F$-spaces.

L. BARIBIAUU, Multifonctions analytiques polygonales.

J. RUSINEK, On generalized canonical commutation relations.

J. K. KOWALSKI, A method of approximation of Besov spaces.

\section{Volume 96, Number 3}

W. CUPAin, On the essential spectrum and eigenvalue asymptotics of certain Schrödinger operators.

M. DIPANr and M. Juncili, On weak $(r, 2)$-summing operators and weak Hilbert spaces.

G. R. Gooluson and M. LiMANCZXK, On the rank of a class of bijective substitutions.

W. HEBrsci and A. SIKORA, A smooth subadditive homogeneous norm on a homogeneous group.

A. FIERNNIJEZ and V. MOHLLER, Renormalizations of Banach and locally convex algebras.

V. MASCONI, Some remarks on the uniform approximation property in Banach spaces.

H. Kamowirz and S. Scuzinbirg, Some properties of endomorphisms of Lipschitz algebras.

W. Heisisch, Almost everywhere summability of eigenfunction expansions associated to elliptic operators. 